\title{
Effects of labeling and number of pretraining exposures on children's perceptual judgments ${ }^{1}$
}

PAUL S. SPEAR ${ }^{2}$ AND NANCY P. WRAY ${ }^{3} D E P A R T M E N T$ OF PSYCIIOLOGY, UNIVERSITY OF DENVER, Denver, Colo. 80210

The effects of verbal labels used during pretraining on subsequent discrimination were investigated in third and fourth grade children. All stimuli presented to an $S$ were identical. Results supported Miller and Dollard's hypothesis of acquired equivalence of cues and only minimally supported Gibson and Gibson's notion that increased practice results in more veridical perception.

In the area of perceptual judgments two quite different theoretical hypotheses are discussed. The Miller \& Dollard (1941) hypothesis of acquired equivalence of cues states that during a pretraining period the association of common verbal labels with visual cues increases the similarity between the stimulus complexes associated with the cues. As a result, Ss making "same-different" judgments to these cues during a test period are more likely to give "same" judgments than if the common labels were not used during the pretraining period. Miller and Dollard also predict that the association of distinctive labels with cues during a pretraining period will decrease the similarity between stimulus complexes associated with the cues. As a result there should be more "different" judgments during the test period than if the distinctive labels were not used during pretraining.

In contrast, the Gibson \& Gibson (1955) hypothesis infers that a pretraining period affects future "same-different" judgments by making cue perception more veridical during the pretraining period. Accordingly increased veridical perception should occur regardless of type of label used during pretraining. Further, veridical judgments should increase with longer pretraining periods.

Although the effects of labeling on perceptual judgments have often been studied (e.g., Arnoult, 1953; Cantor, 1955; Gerjuoy, 1964; Katz, 1963; Norcross \& Spiker, 1957; Reese, 1960; Robinson, 1955) results have been inconsistent. One possible reason for this lack of consistency is that objective differences between stimuli compared have not been controlled across studies.

The present study aims at investigating the effects of labeling on perceptual judgment when objective differences between stimuli being compared are reduced to zero. The use of objectively identical stimuli eliminates the need for accurate measurement of stimulus differences, and facilitates attempts to replicate past findings and to test theoretical positions.

Seventy boys and girls attending third and fourth grades served as Ss and were presented the task by an adult male E. Ten Ss were randomly assigned to each of seven cells in a 3 (pretraining conditions) by 2 (number of exposures during pretraining) factorial design with one additional experimental group. The three pretraining conditions consisted of: (1) Ss receiving one common label (CL), (2) Ss receiving three different labels (DL), and (3) Ss receiving no label (NL). During pretraining Ss received either nine or 27 slide exposures.

The apparatus consisted of a Kodak Carousal 800 slide projector, a shutter attached to a timer controlling duration of slide exposures and intertrial interval, a set of slides, and a tunnel $5 \mathrm{ft}$ long. The tunnel was a 5 -ft cylinder: At one end was the projector and at the other was an eyepiece for $S$ to view the stimuli. The stimuli were projected onto an 8 -sq in. opaque screen located midway in the tunnel.

Each slide was either a single or double image of one of four nonsense forms used by Katz (1963). Each slide with a single image (used during pretraining) and a double image (used during test trials) was exposed for $.77 \mathrm{sec}$. A 10 -sec interval separated slide exposures during both pretraining and test trials. All slides were shown with a focus that gave a very blurred outline of the figure. During both pretraining and test trials, only one of four nonsense forms was used for any given $\mathrm{S}$. Each $\mathbf{S}$ worked individually under instructions that the pictures were of animals found by a space explorer. He was told to look at each picture carefully because, although the pictures looked very much alike, they were of different animals. Immediately prior to each pretraining slide exposure, $\mathrm{E}$ told $\mathrm{Ss}$ in the $\mathrm{CL}$ groups that the animals' names were "Buz." For Ss in the DL groups, E named the animals "Jan" on one-third of the trials, "Buz" on one-third of the trials, and "Ric" on one-third of the trials. Within each block of nine pretraining trials, each of the three labels was represented equally and presented randomly. Ss in the NL groups were told merely to look carefully at each slide. The Ss never overtly verbalized the labels.

At the beginning of the test trials, $S$ was told to press a button marked "same" if the slide showed two pictures of the same animal and to press a button marked "different" if the slide showed pictures of two different animals. He was instructed to make his responses during the interval between slide exposures. Each $\mathrm{S}$ was given 50 double image test trials. Each of the four forms was presented to at least two $S s$ in each of the seven experimental conditions.

Table 1 shows the means and variances of number of "different" judgments (errors) made by each group. Raw data scores indicated that no S in the NL groups gave "same" (veridical) judgments on all 50 test trials.

A 2 by 3 analysis of variance was used to study the effects of number of pretraining trials and type of label (NL, CL, and DL) upon number of "different" (non-veridical) judgments when number of stimulus exposures during pretraining was controlled 19 or 27 trials). Number of stimulus-label pairings was not controlled (i.e.. the number of times a label was attached to a stimulus). For example $\mathrm{Ss}$ in the $\mathrm{CL}-27$ group received 27 trials of one label whereas Ss in the DL-27 group received only nine trials on each of three labels.

The significant main effect of Label $(F=4.64, \mathrm{df}=2 / 54$, $p<.025)$ yielded support for the Miller and Dollard hypothesis of acquired equivalence of cues. The critical difference between two means required for a significant $t$ at the .05 level (Lindquist, $\mathbf{1 9 5 3}$. p. 93) indicated that the number of "same" judgments made by the $C L$ group was significantly larger than that made by either the NL or DL groups. Since the number of "different" judgments made by the DL group was not significantly greater than that made by the NL group, the hypothesis of acquired distinctiveness of cues was not unequivocally supported.

Contrary to Gibson and Gibson's hypothesis of an increase in veridical judgments following pretraining, there was no significant main effect of trials. In fuct the trend toward an increase in non-veridical judgments with increased pretraining is in the opposite direction from that postulated by Gibson and Gibson.

A second analysis was performed to study the effects of number of labels used during pretraining on number of "different" judgments when the number of stimulus-label pairings during

Table 1

Error Means and Variances in the Various Pretraining Groups $\mathrm{N}=10$ per Cell

\begin{tabular}{|c|c|c|c|c|}
\hline \multirow[b]{2}{*}{$\begin{array}{l}\text { Number of pre- } \\
\text { training trials }\end{array}$} & & \multicolumn{3}{|c|}{ Type of label used during pretraining } \\
\hline & & $\begin{array}{c}\text { No } \\
\text { label }\end{array}$ & $\begin{array}{c}\text { Common } \\
\text { label }\end{array}$ & $\begin{array}{c}\text { Different } \\
\text { label }\end{array}$ \\
\hline \multirow{2}{*}{9} & $\overline{\mathrm{X}}$ & 15.30 & 8.40 & 17.80 \\
\hline & $S^{2}$ & 119.78 & 77.60 & 65.73 \\
\hline \multirow{2}{*}{27} & $\overline{\mathrm{X}}$ & 19.90 & 13.90 & 22.00 \\
\hline & $\mathrm{S}^{2}$ & 55.21 & 106.54 & 107.78 \\
\hline \multirow{2}{*}{81} & $\overline{\mathrm{X}}$ & & & 11.30 \\
\hline & $\mathrm{S}^{2}$ & & & 100.46 \\
\hline
\end{tabular}




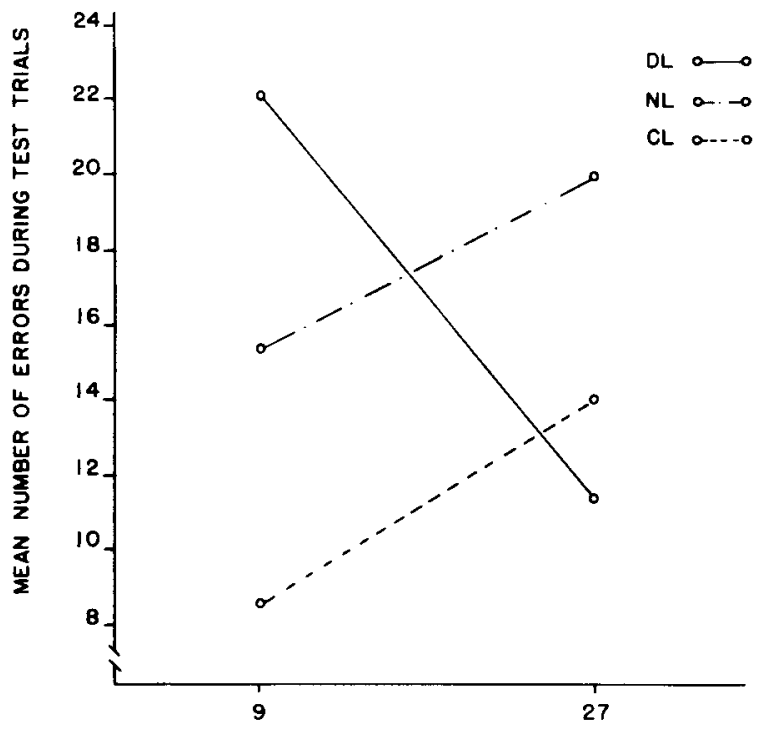

NUMBER OF CUE-LABEL PAIRINGS IN PRETRAINING

Fig. 1. Mean number of "different" judgments (errors) by number of verbal label pairings.

pretraining was controlled. A 3 (type of label) by 2 (number of pairings) analysis of variance compared the test performance of groups which had either none, one, or three labels paired with the identical stimulus forms. A given label was used either 9 or 27 times during pretraining. Total number of pretraining stimulus exposures was not controlled. All groups shown in Table 1 except the DL-9 group were used in the analysis.

The significant Label by Number of Pairings interaction $(F=$ 8.77 , df $=2 / 54, p<.005$ ) shown in Fig. 1 , suggested that the ordering of groups noted with nine label pairings (i.e., number errors in $\mathrm{CL}<\mathrm{NL}<\mathrm{DL}$ ) dissipated with 27 pairings (DL $<\mathrm{CL}<\mathrm{NL}$ ). Further $t$ test analyses (Lindquist, 1953, p. 93) indicated that DL-81 Ss (27 pairings with each of the three labels) made significantly $(\mathrm{p}<.05)$ fewer "different" judgments than DL-27 Ss ( 9 pairings with each of the three labels). In contrast, NL and CL Ss showed nonsignificant trends toward increases in the number of "different" judgments with increased label pairings. In short, the only evidence that could be considered support for the Gibson and Gibson hypothesis is that DL-81 Ss made more veridical judgments than DL-27 Ss.

In order to determine whether pretraining effects were consistent over blocks of test trials, the total number of different judgments were divided into five blocks of 10 trials each. An analysis of variance showed that errors in the first block of trials were not more prevalent than in any other block of trials.

In conclusion, the primary effect of using verbal labels in pretraining seems to be that of making the stimuli either more similar (if identical cues are used) or more dissimilar (if different cues are used), thus lending support to Miller \& Dollard's position (1941). Gibson and Gibson's notion that pretraining involving repeated exposure to stimuli results in more veridical judgment was not generally upheld. In interpreting the results it must be kept in mind that the stimuli exposed to any one $S$ were identical. One possible reason why the effects of acquired distinctiveness of cues dissipated in the DL-81 group is that with increased exposure to stimuli, $S$ may clearly perceive them to be identical regardless of the different verbal labels. Another possible explanation may be that, initially in pretraining, learning different labels to similar or identical stimuli may result in a greater perceived difference between them, but as trials increase, the acquired distinctiveness of cues decreases and the effect of prolonged pretraining results in more veridical perception according to Gibson and Gibson's notion.

\section{REFERENCES}

ARNOULT, M. D. Transfer of predifferentiation training in simple and multiple shape discrimination. J. exp. Psychol., 1953, 45, 401-409.

CANTOR, G. N. Effects of three types of pretraining on discrimination learning in preschool children. J. exp. PsychoL, 1955, 49, 339-342.

GERJUOY, I. R. Discrimination learning as a function of the similarity of the stimulus names. Child Develpm., 1964, 35, 677-684.

GIBSON, J. J., \& GIBSON, E. J. Perceptual learning: Differentiation or enrichment? Psychol Rev., 1955, 62, 32-41.

KATZ, P. A. Effects of labels on children's perception and discrimination learning. J. exp. Psychol., 1963, 66, 423-428.

LINDQUIST, E. F. Design and analysis of experiments in psychology and education. Boston: Mifflin, 1953.

MILLER, N. E., \& DOLLARD, J. Social leaming and imitation. New Haven: Yale University Press, 1941.

NORCROSS, K. J., \& SPIKER, C. C. The effects of type of stimulus pretraining on discrimination performance in preschool children. Child Develpm, 1957, 28, 79-84.

REESE, H. W. Motor paired-associate learning and stimulus pretraining. Child Develpm, 1960, 31, 505-513.

ROBINSON, G. S. The effects of learning verbal labels for stimuli on their later discrimination. J. exp. Psychol., 1955, 49, 112-114. NOTES

1. The authors are grateful to Mr. Harry J. Van Zytveld and the staff of Van Dellen Christian School, Denver, Colorado, for supplying the Ss and experimental space required for execution of the study.

2. This research was supported in part by USPHS Traineeship MH-8297, to the first author. Now at San Diego State College, San Diego, California. 3. Now at St. Louis University, St. Louis, Missouri. 